\title{
A study of rumors spreading model with on scale-free network
}

\author{
Tao $\mathrm{Li}^{1,2}$, Qian Zhang ${ }^{2}$ \\ ${ }^{1}$ Basic Courses Department, Ordnance Engineering College, Shijiazhuang 050003, China; \\ 2 The 260 hospital of PLA, Shijiazhuang 050043, China. \\ leo_119@163.com
}

Keywords: Scale-Free network, rumors, time delay nonlinear infectivity, basic reproductive number.

\begin{abstract}
In this paper, a novel SIRS model of rumors on scale-free network with time delay is proposed. The basic reproductive number for the model is presented. We prove that the rumors will die out when the basic reproductive number is less than the unity. Whereas the permanence of the rumors is shown if the basic reproductive number exceeds the unity. Numerical simulations confirmed the analytical results.
\end{abstract}

\section{Introduction}

Rumors are nearly as old as human history, but with the rise of the Internet, they have become ubiquitous. In the new media age, social rumor that spread through the Internet has become a typical public problem in the field of public administration. There are often times when destructive rumors can cause social panic and even huge economic loss [1]. As such, governing of internet rumors is a common challenge confronting the international community at present. As China is entering a critical period of social transition, increasing social conflicts call for an effective governance to prevent the spreading of online rumors from threatening social stability. The study of rumor spreading has important theoretical as well as practical implications.

A classic rumor model is the DK model proposed by Daley and Kendal in 1965 [2]. In their model, the population of interest is divided into three groups: people who know and spread the rumor, people who do not know the rumor and people who know but do not transmit the rumor. Then Maki and Thomson [3] modified the DK model into the MT model, which assumes that a spreader changes to a stifler (who knows but does not transmit the rumor) once s/he contacts another spreader.

Based on these early models, many researchers carried on the study of rumor spreading [4] and related it to the topological properties of social networks [5]. Besides, Zanette [6] studied the rumor spreading model on small-world networks and found the existence of the critical threshold for rumor spreading. Moreno et al. [7] examined the dynamics of rumor spreading on scale-free networks. Isham et al. [8] analyzed the final distribution size of rumors on general networks.

However, most previous works mainly focus on the impact of the underlying topology and assume that the transmission is uniformly distributed among all links, that is, each individual will try to contact all its neighbors once within one time step. In fact, this kind of uniform transmission is induced from the assumption that each node's potential infectivity, counted by its possible maximal contribution to the propagation process within one time step, is strictly equal to its degree. However, there are still many real spreading processes, which cannot be rightly characterized by this assumption . In epidemic contact networks, the super-spreader has many acquaintances; however, he/she could not contact all his/her acquaintances within one time step .

In this paper, we aim to overlook the above defects and understand the spreading behaviors of rumors in Internet. We present a novel SIRS epidemic model of rumors to investigate the impact of nonlinear infectivity in Internet with the scale-free property. We obtain the corresponding spreading threshold and analyze the globally dynamic behaviors of the rumors. Then, the numerical simulations are given, which are well consistent with the theoretical results.

The remainder of this paper is structured as follows. In Section 2, we will briefly formulate this novel epidemic model. Then in Section 3, we determine the threshold value and analyze the globally 
dynamic behaviors of the rumors. In Section 4, we carry out extensive numerical simulations to verify the theoretical analysis in Section 3. At last, in Section 5 we summarize this work.

\section{Model formulation}

It is well known [9] that the node degrees of Internet asymptotically follow a power law distribution, $P(k) \square k^{-\gamma}$, where $P(k)$ stands for the probability that a node chosen randomly from Internet is of degree $k$. For this model in Internet, each individuals is represented by a vertex of the network and the edges are communication links between them, along which the rumors may spread. Furthermore, the nodes can only exist in three discrete states, i.e., susceptible (S), infected (I) and removed. Susceptible ones are those who have never heard of the rumor and can be easily infected by the rumor with probability $\lambda(k)$. Infected ones comprise individuals who have heard of the rumor and will spread it to others with probability $p$. A infected individual may forget the rumor and switch his state to susceptible after time $\tau$. $\tau$ is the average infected period. Removed ones are those will not spread the rumor any more. The removed individual turns into a susceptible one with probability $\mu$. Let $S_{k}(t), I_{k}(t)$ and $R_{k}(t)$ be the densities of susceptible, infected, and removed vertexes of degree $k$ at time $t$. Obviously, they must satisfy the normalized condition $S_{k}(t)+I_{k}(t)+R_{k}(t)=1$ if the total number of the node is fixed. All parameters are positive. Then by applying the mean-field technique to the above assumptions, we have the following dynamics model based on delay differential equations

$$
\left\{\begin{array}{l}
\frac{d S_{k}(t)}{d t}=-\lambda(k) S_{k}(t) \Theta(t)+\mu R_{k}(t) \\
\frac{d I_{k}(t)}{d t}=p \lambda(k) S_{k}(t) \Theta(t)-p \lambda(k) S_{k}(t-\tau) \Theta(t-\tau) \\
\frac{d R_{k}(t)}{d t}=(1-p) \lambda(k) S_{k}(t) \Theta(t)+p \lambda(k) S_{k}(t-\tau) \Theta(t-\tau)-\mu R_{k}(t)
\end{array}\right.
$$

$\Theta(t)$ denotes the probability from any given link to an infected node. It satisfies the relation

$$
\Theta(t)=\frac{\sum_{k=1}^{n} \varphi(k) P(k) I_{k}(t)}{\langle k\rangle}
$$

$\varphi(k)$ denotes the nonlinear infectivity related with the degree of an infected node, where $\varphi(k)=\alpha k^{\alpha} / 1+b k^{\alpha}, 0 \leq \alpha \leq 1, a>0, b \geq 0[15] .\langle k\rangle$ is the average degree within the network.

\section{The analysis of the model}

Let

$$
R_{0}=\frac{p \tau\langle\lambda(k) \varphi(k)\rangle}{\langle k\rangle}
$$

Theorem 1. There is always a rumors-free equilibrium $E_{0}\{(1,0,0)\}_{k}$ for system (1) and when $R_{0}<1$; system (1) has a unique endemic equilibrium $E_{*}\left(S_{k}^{*}, I_{k}^{*}, R_{k}^{*}\right), S_{k}^{*}, I_{k}^{*}, R_{k}^{*}$ denote the densities of susceptible, infected, and quarantined vertexes of degree $k(k=1,2, \cdots n)$ respectively when the system arrives at a stationary state.

Proof. It is easily verified that $E_{0}$ is always an equilibrium of system (1).

Transform the third equation of system (3) into the following integral form

$$
I_{k}(t)=p \lambda(k) \int_{t-\tau}^{t} S_{k}(u) \Theta(u) d u
$$


To get the equilibrium solution $E_{*}$ we need to make the right side of system (1) equal to zero. Then the equilibrium $E_{*}$ should satisfy

$$
\left\{\begin{array}{l}
I_{k}^{*}=p \lambda(k) \tau S_{k}^{*} \Theta^{*} \\
\mu R_{k}^{*}=\lambda(k) S_{k}^{*} \Theta^{*} \\
I_{k}^{*}+S_{k}^{*}+R_{k}^{*}=1
\end{array}\right.
$$

where

$$
\Theta^{*}=\frac{1}{\langle k\rangle} \sum_{k} P(k) \varphi(k) I_{k}^{*}
$$

Solving Eq.(4) yields

$$
I_{k}^{*}=\frac{p \tau \mu \lambda(k) \Theta^{*}}{\lambda(k) \Theta^{*}+p \tau \mu \lambda(k) \Theta^{*}+\mu}
$$

Substituting Eq.(6) into Eq.(5), we can obtain the self-consistent equation about $\Theta^{*}$ as follows

$$
\Theta^{*}=\frac{1}{\langle k\rangle} \sum_{k} P(k) \varphi(k) I_{k}^{*}=\frac{1}{\langle k\rangle} \sum_{k} P(k) \varphi(k) \frac{p \tau \mu \lambda(k) \Theta^{*}}{\lambda(k) \Theta^{*}+p \tau \mu \lambda(k) \Theta^{*}+\mu}=f\left(\Theta^{*}\right)
$$

Apparently, $\Theta^{*}=0$ is always a trivial solution, which denotes the rumors-free state. Therefore, a nontrivial solution exists provided

$$
f\left(\Theta^{*}\right)=\frac{1}{\langle k\rangle} \sum_{k=1}^{n} k \varphi(k) P(k) \frac{\sigma \lambda \Theta^{*}}{\sigma \mu+\lambda k \Theta^{*}}=0
$$

Since

$$
\frac{d f\left(\Theta^{*}\right)}{d \Theta^{*}}>0, f(1)>0
$$

a nontrivial solution exists if and only if $f(0)<0$, i.e., $R_{0}>1$.

Substituting the nontrivial solution of $f(\Theta)=0$ into Eq.(6), we can get $I_{k}^{*}$. By Eq.(4) and (6), we can easily obtain that

$0<S_{k}^{*}<1,0<I_{k}^{*}<1,0<R_{k}^{*}<1$

Therefore, the equilibrium $E^{*}$ is well-defined. Hence, when $R_{0}>1$, one and only one endemic equilibrium of system (1) exists. This completes the proof.

\section{Numerical simulation}

Extensive numerical simulations are carried out on BA scale-free model to demonstrate the above mentioned theorems. The degree distribution is $P(k)=c k^{-\gamma}$, and constant $c$ satisfies $\sum_{k=1}^{n} P(k)=1$. Here we set the maximum degree $n=100$, the minimum degree $m=1$.

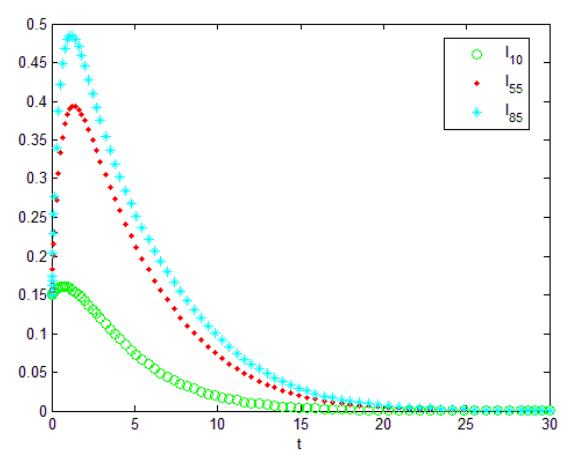

Figure 1: Evolutions of $I_{10}(t), I_{55}(t), I_{85}(t)$, for the system (1) with condition $R_{0}<1$.

Consider system (1) with parameters $\lambda=0.2, \mu=0.3, \tau=2$, $p=0.2, a=6, b=1, \alpha=0.6$, which runs 
on a scale-free network with $\gamma=2.5$. Then $R_{0}=0.453<1$. For a set of initial conditions satisfying $I_{k}(0)=0.001, S_{k}(0)=0.999, R_{k}(0)=0$, Fig. 1 shows the evolutions of $I_{10}(t), I_{55}(t), I_{85}(t)$, from which it can be seen that rumors will tend to extinction, in agreement with Theorem 1 .

Consider system (1) with parameters $\lambda=0.5, \mu=0.3, \tau=2, \sigma=0.2, a=6, b=1, \alpha=0.6$, which runs on a scale-free network with $\gamma=2.5$. Then $R_{0}=1.373>1$. For a set of initial conditions satisfying $I_{k}(0)=0.001, S_{k}(0)=0.999, R_{k}(0)=0$, Fig. 2 shows the evolutions of $I_{10}(t), I_{55}(t), I_{85}(t)$, from which it can be seen that rumors will tend to extinction, in agreement with Theorem 1.

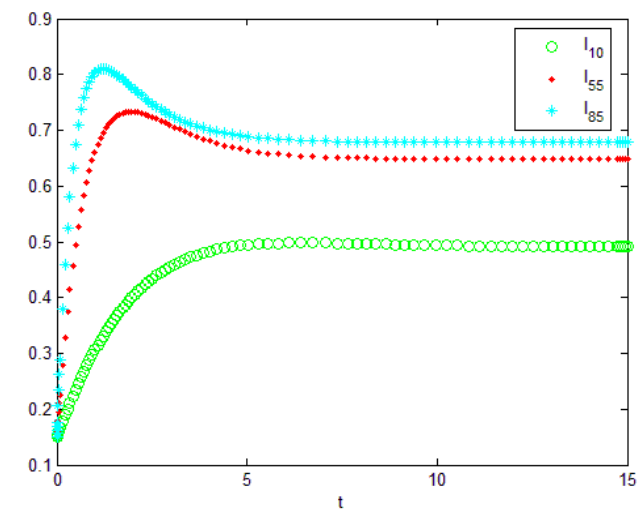

Figure 2: Evolutions of $I_{10}(t), I_{55}(t), I_{85}(t)$, for the system (1) with condition $R_{0}>1$.

\section{Conclusion}

In summary, to better understand the effect of the time delay on the spread of rumors, we introduce a novel SIRS model capturing the epidemics of rumors in Internet with scale-free networks property which includes the nonlinear infectivity and infection delay. The spreading threshold for the model has been determined. These results help to work out policies of inhibiting rumors.

\section{References}

[1] S.A. Thomas, Lies, damn lies, and rumors: an analysis of collective efficacy, rumors, and fear in the wake of Katrina, Sociol. Spectrum 2007, 27: 679-703.

[2] D.J. Daley, D.G. Kendall, Epidemics and rumours, Nature. 1964, 204: 1118.

[3] D. Maki, M. Thomson, Mathematical Models and Applications, Prentice-Hall, Englewood Cliffs, 1973.

[4] C. Lefevre, P. Picard, Distribution of the final extent of a rumor process, J. Appl. Probab. 1994, 31: 244-249.

[5] B. Doerr, M. Fouz, T. Friedrich, Social networks spread rumors in sublogarithmic time, Electron. Notes Discrete Math. 2011, 38: 303-308.

[6] D.H. Zanette, Dynamics of rumor propagation on small-world networks, Phys. Rev. E. 2002, 65: 041908.

[7] Y. Moreno, M. Nekovee, A. Pacheco, Dynamics of rumor spreading in complex networks, Phys. Rev. E. 2004, 69: 066130.

[8] V. Isham, S. Harden, M. Nekovee, Stochastic epidemics and rumours on finite random networks, Physica A. 2010, 389: 561-576.

[9] M. Faloutsos, P. Faloutsos, C. Faloutsos, On power-law relationships of the Internet topology, in: ACM SIGCOMM Comput. Commun. Rev. 1999, 29(4): 251-262. 